\title{
Composition of the nuclear periphery from antiproton absorption using short-lived residual nuclei
}

\author{
R. Schmidt, F. J. Hartmann, B. Ketzer, and T. von Egidy \\ Physik-Department, Technische Universität München, D-85747 Garching, Germany
}

T. Czosnyka, J. Jastrzębski, M. Kisieliński, P. Lubiński, P. Napiorkowski, L. Pieńkowski, and A. Trzcińska

Heavy Ion Laboratory, Warsaw University, PL-02-093 Warsaw, Poland

B. Kłos

Physics Department, Silesian University, PL-40-007 Katowice, Poland

R. Smolańczuk and S. Wycech

Soltan Institute for Nuclear Studies, PL-00-681 Warsaw, Poland

W. Pöschl

Department of Physics, Duke University, Durham, North Carolina 27708-0305

K. Gulda and W. Kurcewicz

Institute of Experimental Physics, Warsaw University, PL-02-093 Warsaw, Poland

E. Widmann

CERN, CH-1211 Geneva 23, Switzerland

(Received 23 March 1999; published 1 October 1999)

\begin{abstract}
The neutron-to-proton density ratio at the nuclear periphery was determined for six targets from the yield of nuclei with mass $\left(A_{t}-1\right)$ generated by antiproton annihilation on targets with mass number $A_{t}$. A new setup allowed us to measure this yield for nuclei with half-lives down to $6 \mathrm{~s}$. The experiment confirmed the negative correlation between the peripheral neutron-to-proton density ratio and the neutron binding energy, which had been previously found. Results of Hartree-Fock-Bogoliubov and relativistic Hartree-Bogoliubov calculations show good agreement for a large number of nuclei. By comparing the measured values with those derived from Hartree-Fock-Bogoliubov calculations, the ratio $\mathcal{R}$ of the imaginary parts of the effective scattering lengths of antiprotons on bound neutrons and protons was deduced to be $\mathcal{R}=0.69(4)$. For the first time yields of several excited states of some $\left(A_{t}-1\right)$ nuclei have been investigated. [S0556-2813(99)04410-6]
\end{abstract}

PACS number(s): 21.10.Gv, 25.43.+t

\section{INTRODUCTION}

Experiments on the antiproton-nucleus interaction may be seen under two aspects: On the one hand the scattering of fast antiprotons on nuclei, the measurement of level widths and shifts in antiprotonic atoms, and the investigation of residual nuclei after the annihilation may render insight into details of the antiproton-nucleon forces in nuclear matter. On the other hand the reaction of antiprotons with nuclei yields a method to investigate the nucleon density if the peculiarities of nuclear antiproton capture are sufficiently well known [1].

In a stopped $\bar{p}$ experiment the antiprotons are slowed down in the target and finally captured by the target atoms into atomic orbits with large principal quantum number $n$. They cascade down the bound levels under emission of $\mathrm{Au}-$ ger electrons and $\mathrm{x}$ rays [2]. When they reach states with low $n$ or low angular momentum, the overlap of the antiprotonicatom wave function and the nucleus becomes sizable and the strong interaction between antiproton and nucleus widens and shifts the atomic levels, thus also influencing the energies of the corresponding transition $\mathrm{x}$ rays. These widths and shifts were studied in various experiments (e.g., [3-7]). The antiproton annihilation takes place in a nuclear region far outside the half-density radius [8]. This makes the antiproton a good tool to probe the nuclear periphery. The antiprotons are annihilated when they get into contact either with a neutron or a proton. In most cases the mesons produced during the annihilation initiate an intranuclear cascade that results in the prompt emission of particles $\left(n, p, d, t,{ }^{3} \mathrm{He},{ }^{4} \mathrm{He}, \ldots\right)$, in evaporation, and sometimes even in fission or fragmentation of the target nucleus.

In preceding experiments the mass distribution of residual nuclei after the annihilation process was investigated [9-11]. It was found that after annihilation on a target with mass $A_{t}$ a substantial number of residual nuclei remains with mass $\left(A_{t}-1\right)$. The investigation of these residual nuclei with either one neutron or one proton fewer than the target nucleus yields information on the neutron-to-proton density ratio in the annihilation region [12]. The measured ratios may be compared with the predictions of different nuclear models [8].

The antiproton usually annihilates in a region which is much more peripheral than those investigated by most of the 
other methods, such as elastic scattering, nuclear reactions, and $\mathrm{x}$ rays from pionic and kaonic atoms [13]. Furthermore, the process can differentiate between neutrons and protons. This selectivity was first investigated by the measurement of meson multiplicities after antiproton annihilation [14]. The method used in our experiment, which is sensitive to a region even farther away from the nuclear center than average antiproton annihilation, was first introduced in Ref. [12]. In subsequent experiments the neutron-to-proton density ratio has been determined for 13 nuclei $[15,16]$. In the present experiment six more nuclei were investigated, with the smallest half-life of the residual $\left(A_{t}-1\right)$ nuclei as low as $6 \mathrm{~s}$. The measurements provide an additional and essential systematic investigation of the nuclear periphery.

\section{EXPERIMENTAL METHOD}

In the outer nuclear region, where antiprotons are annihilated, the nucleon density is less than $10 \%$ of the density inside the nucleus. The antiprotons are annihilated essentially on one nucleon, but the mesons produced in this process can initiate a multinucleon reaction. The probability for annihilation is proportional to the single-nucleon densities. The annihilation rate is described by an effective-length parameter $\operatorname{Im}\left(a_{N}\right)$, while the range of the annihilation is usually taken to follow the nuclear density distribution [17]. Thus the total absorption rate $W$ becomes

$$
W=\frac{4 \pi}{\mu_{N \bar{p}}} \operatorname{Im}\left(a_{N}\right) \int d \vec{R} \widetilde{\rho}_{N}(\vec{R})\left|\Psi_{\bar{p}}(\vec{R})\right|^{2}, \quad N=n, p .
$$

Here $\widetilde{\rho}_{N}(\vec{R})$ is the nucleon density folded with a form factor, which represents the range of the single-nucleon annihilation, and $\Psi_{\bar{p}}(\vec{R})$ is the atomic wave function of the antiproton [8]. $\mu_{N \bar{p}}$ and $a_{N}$, finally, are the reduced mass and the strong-interaction effective scattering length, respectively.

Since the effective scattering length is supposed to be independent of nuclear properties, the ratio of the absorption probabilities is proportional to the neutron-to-proton density ratio in the region where the annihilation takes place. The radial distribution of these probabilities in heavy nuclei depends only weakly on the quantum numbers $(n, l)$ of the level at which the antiproton is absorbed: for high $l$ the centrifugal barrier prevents the wave function from penetrating into the nucleus; for low $n$ and $l$ the wave function is strongly damped inside the nucleus due to the strong interaction $[16,18]$.

In most cases pions which are produced during annihilation interact with the nucleus. If the annihilation takes place sufficiently far away from the nuclear center, the probability is high, however, that all pions miss the residual nucleus and the nucleus remains cold. This fact makes the method sensitive to a region about $1 \mathrm{fm}$ outside the average antiproton absorption region, i.e., about $3 \mathrm{fm}$ outside the mean nuclear radius. The residual nucleus has one proton fewer than the target nucleus if the annihilation took place with a proton or one neutron fewer if the annihilation took place with a neutron.
Excitations of the residual nucleus due to antiproton absorption on deeply bound nucleons have to be taken into account. Annihilations with these nucleons, which occupy orbits with binding energies larger than the binding energy of the last neutron, could produce residual nuclei with more than one nucleon missing from the target nucleus. Fortunately, this results in corrections of less than 15\% [8].

The probability to produce a cold product with mass $\left(A_{t}\right.$ -1) was calculated using the optical potential from [19]. The probability distribution has a maximum about $3 \mathrm{fm}$ outside the half-density radius $R_{1 / 2}$ of the nucleus (see Fig. 10 of Ref. [16]) and its radial extension [full width at half maximum (FWHM)] is between 2 and $3 \mathrm{fm}$. With a number $N(\bar{p}, n)$ of cold reaction products with one neutron fewer, and a number $N(\bar{p}, p)$ of those with one proton fewer than the target nuclei the ratio of the yields of the residual nuclei is

$$
\frac{N(\bar{p}, n)}{N(\bar{p}, p)}=\frac{\operatorname{Im}\left(a_{n}\right)}{\operatorname{Im}\left(a_{p}\right)} \frac{\int \widetilde{\rho}_{n}(\vec{R})\left|\Psi_{\bar{p}}(\vec{R})\right|^{2} P_{\text {miss }, n}(\vec{R}) P_{\mathrm{dh}, n}(\vec{R}) d \vec{R}}{\int \widetilde{\rho}_{p}(\vec{R})\left|\Psi_{\bar{p}}(\vec{R})\right|^{2} P_{\text {miss }, p}(\vec{R}) P_{\mathrm{dh}, p}(\vec{R}) d \vec{R}} .
$$

Here $P_{\mathrm{dh}, N}(\vec{R})$ and $P_{\text {miss, } N}(\vec{R})$ are corrections for the annihilaton with a deeply bound nucleon and the probability that the pions produced at $\vec{R}$ miss the nucleus, respectively [8]. The peripheral halo factor $f_{\text {halo }}^{\text {periph }}$ may then be defined [16] as

$$
f_{\text {halo }}^{\text {periph }}:=\frac{N(\bar{p}, n)}{N(\bar{p}, p)} \frac{Z_{t}}{N_{t}} \frac{\operatorname{Im}\left(a_{p}\right)}{\operatorname{Im}\left(a_{n}\right)} \approx \frac{\rho_{n}\left(R_{1 / 2}+3 \mathrm{fm}\right)}{\rho_{p}\left(R_{1 / 2}+3 \mathrm{fm}\right)} \frac{Z_{t}}{N_{t}} .
$$

The most widely used value for $\operatorname{Im}\left(a_{n}\right) / \operatorname{Im}\left(a_{p}\right)$ is $0.63(6)$ $[14,20] . \quad \operatorname{Im}\left(a_{n}\right) / \operatorname{Im}\left(a_{p}\right)=0.82(4) \quad$ and $\quad \operatorname{Im}\left(a_{n}\right) / \operatorname{Im}\left(a_{p}\right)$ $=0.47(4)$ were deduced from experiments on the emission of charged particles after antiproton annihilation with deuterons [21] and helium nuclei [22], respectively. The total number of products with mass $\left(A_{t}-1\right)$ depends on the nature of the antiproton-nucleus interaction, the size and geometry of the target nucleus, the pion-nucleus interaction, the energy distribution of the produced pions, and their kinematic correlations. This made theoretical predictions rather scarce. The only calculation performed agrees with the measurement well within the accuracy quoted by the authors [16].

If the residual nuclei with mass $\left(A_{t}-1\right)$ are unstable and their decay is followed by the emission of $\gamma$ rays, the absolute number of these nuclei can be determined by $\gamma$ spectroscopy [12]. In Ref. [16] residual nuclei with half-lives larger than 5 min were investigated. In the experiment described in this publication we were able to extend the measurements to nuclei with half-lives down to $6 \mathrm{~s}$ by employing a fast target transport system. This allowed us to add several interesting nuclei to the systematic investigation: the doubly magic ${ }^{48} \mathrm{Ca} ;{ }^{112} \mathrm{Sn}$, and ${ }^{124} \mathrm{Sn}$ with magic proton number, and also ${ }^{100} \mathrm{Mo},{ }^{104} \mathrm{Ru}$, and ${ }^{116} \mathrm{Cd}$. For the two last neutron-rich nuclei the corresponding neutron-deficient isotopes ${ }^{96} \mathrm{Ru}$ and ${ }^{106} \mathrm{Cd}$ had already been measured [16]. 


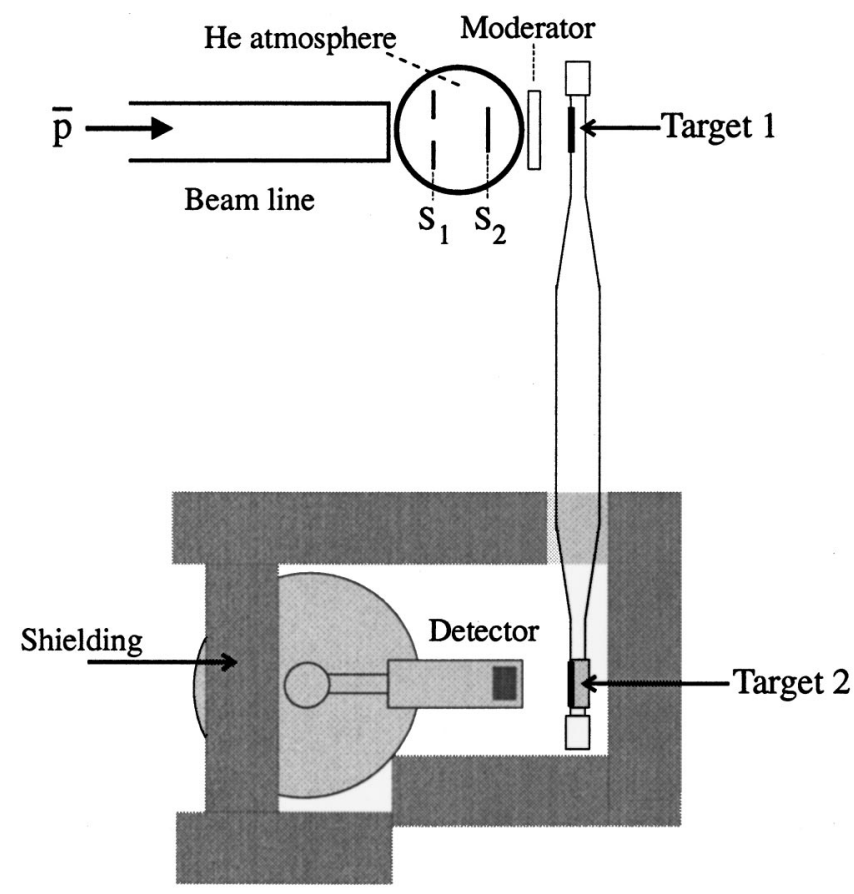

FIG. 1. Schematic view of the setup for irradiation and activation measurement of short-lived isotopes. $S_{1}$ and $S_{2}$ are anticounter and counter of the scintillation-counter telescope, respectively.

\section{EXPERIMENTAL SETUP}

The experiment was performed with the antiproton beam provided by the LEAR facility of CERN. The initial momentum of the antiprotons was $106 \mathrm{MeV} / c$. They were detected by a telescope counter which was placed inside a vessel filled with He. This was necessary because antiprotons with such a small energy $(6.0 \mathrm{MeV})$ have insufficient range in air to reach the target. A degrader of $50 \mu \mathrm{m}$ Mylar was placed behind the vessel in order to let all antiprotons stop well inside the target (cf. Fig. 1). The antiproton range and beam diameter were checked with a series of probes of different thicknesses and diameters at the target position.

The targets were between 12 and $30 \mathrm{mg} / \mathrm{cm}^{2}$ thick (cf. Table I). They were placed on a transport system which moved them within $2.3 \mathrm{~s}$ out of the beam line and into a position in front of a HPGe detector. This detector (relative efficiency 26\%) was housed in a hut shielded with lead and paraffin in order to suppress the background from the beam. While the activity of one target was measured the other one was irradiated and vice versa. Very much like with a funiculaire, the targets moved on two rails, which ensured that both had the same end positions, but allowed them to pass each other during the transport. As some of them were rather fragile they were slowly decelerated before stopping in order to reach the end position smoothly. The targets were irradiated for two to three half-lives of the nucleus of interest, moved to the measuring place, measured for the same length of time, moved back into the in-beam position, irradiated again, and so on. Each target was exposed to the $\bar{p}$ beam for $3-6 \mathrm{~h}$; this resulted in a total of several times $10^{8}$ antiproton annihilations in each target. During the experiment the pulse height of the amplified detector signal was recorded for each event together with the cumulated number of $\bar{p}$ signals from the telescope counter, the elapsed time in units of $10 \mathrm{~ms}$, and information on the position of the two targets. A pulser with frequency $11 \mathrm{~Hz}$ was used for dead-time correction. In order to get an absolute normalization, an Al target was used as the second target in the setup for all six nuclides. The number of ${ }^{24} \mathrm{Na}$ nuclei produced per antiproton stop in $\mathrm{Al}, N_{\mathrm{Na}}$, had been measured before [16] to be $N_{\mathrm{Na}}=(19.5 \pm 1.0) \times 10^{-3}$. This value was used to determine the absolute number of antiprotons stopped in our targets. After the experiment the activity of the targets was recorded outside the beam area for at least three half-lives of the longest-lived $\left(A_{t}-1\right)$ product.

The half-lives of the investigated isotopes range from $6 \mathrm{~s}$ to 129 days (cf. Table II). The $\gamma$ rays were recorded between $2.3 \mathrm{~s}$ after the end of the irradiation (the minimum delay time introduced by the target transport) and 300 days. In order to deduce the abundance of the residual nuclides, the measured number of characteristic $\gamma$ rays from their decay products was corrected for detector efficiency, decay of the nuclide, $\gamma$-transition probability per decay, as taken from the latest Nuclear Data Sheets and the Brookhaven database [23], and for the enrichment of the isotope in the target. The time dependence of the antiproton beam intensity, to which the production rate of the $\left(A_{t}-1\right)$ nuclei is proportional, was also taken into account. Usually one $\left(A_{t}-1\right)$ nucleus is $\beta^{+}$ or $\beta^{-}$unstable and decays into the other $\left(A_{t}-1\right)$ nucleus under investigation. Therefore the decay of the mother nuclei was also taken into account. A set of six HPGe detectors was used to perform the long-time measurements. A few of the $\gamma$ lines were contaminated by lines from other residual nuclei or from the surroundings. In these cases the intensity of the contamination was determined from other lines of the respective nuclide.

TABLE I. Targets.

\begin{tabular}{lccc}
\hline \hline Target & $\begin{array}{c}\text { Enrichment } \\
(\%)\end{array}$ & $\begin{array}{c}\text { Thickness of the target } \\
\text { material }\left(\mathrm{mg} / \mathrm{cm}^{2}\right)\end{array}$ & $\begin{array}{c}\text { Chemical form } \\
\text { of the target }\end{array}$ \\
\hline${ }^{48} \mathrm{Ca}$ & 14.0 & Carbonate \\
${ }^{100} \mathrm{Mo}$ & 96.8 & 24.7 & Metal \\
${ }^{104} \mathrm{Ru}$ & 95.9 & 29.7 & Powder with $4.2 \mathrm{mg} / \mathrm{cm}^{2}$ glue \\
${ }^{112} \mathrm{Sn}$ & 99.1 & 26.4 & Metal \\
${ }^{116} \mathrm{Cd}$ & 98.9 & 24.7 & Metal \\
${ }^{124} \mathrm{Sn}$ & 93.0 & 28.8 & Metal \\
\hline \hline
\end{tabular}


TABLE II. Number of antiprotons stopped in each target, $\left(A_{t}-1\right)$ nuclei with $Z^{\prime}=Z_{t}-1$ and with $N^{\prime}$ $=N_{t}-1$, half-lives $T_{1 / 2}$, and $\gamma$ energies $E_{\gamma}$ of lines which were used for the evaluation.

\begin{tabular}{|c|c|c|c|c|}
\hline Target & $\begin{array}{c}\text { Number of } \\
\text { stopped } \bar{p}\left(10^{8}\right)\end{array}$ & $\begin{array}{c}Z^{\prime} \text { and } N^{\prime} \\
\text { nucleus }\end{array}$ & $T_{1 / 2}$ & $\begin{array}{c}E_{\gamma} \\
(\mathrm{keV})\end{array}$ \\
\hline \multirow[t]{2}{*}{${ }^{48} \mathrm{Ca}$} & \multirow[t]{2}{*}{8.3} & ${ }^{47} \mathrm{~K}$ & $17.5 \mathrm{~s}$ & $2013,586,565$ \\
\hline & & ${ }^{47} \mathrm{Ca}$ & $4.54 \mathrm{~d}$ & $1297,489,807$ \\
\hline \multirow[t]{2}{*}{${ }^{100} \mathrm{Mo}$} & \multirow[t]{2}{*}{8.3} & ${ }^{99} \mathrm{Nb}$ & $15.0 \mathrm{~s} / 2.6 \mathrm{~m}$ & $138 / 254,351, \ldots$ \\
\hline & & ${ }^{99} \mathrm{Mo}$ & $65.9 \mathrm{~h}$ & $740,181, \ldots$ \\
\hline \multirow[t]{2}{*}{${ }^{104} \mathrm{Ru}$} & \multirow[t]{2}{*}{4.2} & ${ }^{103} \mathrm{Tc}$ & $54.2 \mathrm{~s}$ & $346,136, \ldots$ \\
\hline & & ${ }^{103} \mathrm{Ru}$ & $39.26 \mathrm{~d}$ & 497,610 \\
\hline \multirow[t]{2}{*}{${ }^{112} \mathrm{Sn}$} & \multirow[t]{2}{*}{9.3} & ${ }^{111} \mathrm{In}$ & $7.7 \mathrm{~m} / 2.80 \mathrm{~d}$ & $537 / 245$ \\
\hline & & ${ }^{111} \mathrm{Sn}$ & $35.3 \mathrm{~m}$ & $762,457, \ldots$ \\
\hline \multirow[t]{2}{*}{${ }^{116} \mathrm{Cd}$} & \multirow[t]{2}{*}{3.9} & ${ }^{115} \mathrm{Ag}$ & $18.0 \mathrm{~s} / 20.0 \mathrm{~m}$ & $389,361,113 / 213,472, \ldots$ \\
\hline & & ${ }^{115} \mathrm{Cd}$ & $2.23 \mathrm{~d} / 44.6 \mathrm{~d}$ & $336,528 / 934,1290$ \\
\hline \multirow[t]{2}{*}{${ }^{124} \mathrm{Sn}$} & \multirow[t]{2}{*}{8.5} & ${ }^{123} \mathrm{In}$ & $5.98 \mathrm{~s} / 47.8 \mathrm{~s}$ & $1130,1019 / 126$ \\
\hline & & ${ }^{123} \mathrm{Sn}$ & $40.1 \mathrm{~m} / 129.2 \mathrm{~d}$ & $160 / 1089$ \\
\hline
\end{tabular}

\section{EXPERIMENTAL RESULTS}

The results of the activity measurements are presented in Table III. The peripheral halo factor was determined from

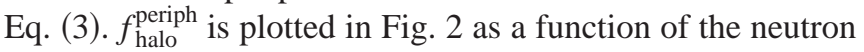
binding energy $B_{n}$ of the respective target nucleus, together with the data from Ref. [16]. The new data confirm the negative correlation of $f_{\text {halo }}^{\text {periph }}$ with $B_{n}$. This shows the crucial role of $B_{n}$ in the development of the peripheral neutron halo in heavy nuclei. The coefficient for the linear correlation between $f_{\text {halo }}^{\text {periph }}$ and $B_{n}$ was found to be $r=-0.79$, the sign for a strong anticorrelation between the two quantities. The large error found for ${ }^{116} \mathrm{Cd}$ is due to the poorly known absolute probability for $\gamma$-ray emission after the decay of the $\left(A_{t}\right.$ -1) residual nuclei in this target. The larger error of the absolute yields as compared to the yield ratios is caused by the additional uncertainty inferred by the normalization. Charge-exchange reactions which could influence the measured ratio of $N(\bar{p}, n) / N(\bar{p}, p)$ were not taken into account as their effect is negligible [16].

The fact that $f_{\text {halo }}^{\text {periph }} \gg 1$ for most of the targets indicates that the neutron density is strongly enhanced in the nuclear periphery as compared with the proton density. Only a few of the investigated nuclei, the proton-rich ones, exhibit an enhanced proton density in the outer nuclear region.

For each target the total yield of nuclei with mass $\left(A_{t}\right.$
-1) was determined besides $f_{\text {halo }}^{\text {periph }}$. This yield is close to $10 \%$ for the newly investigated nuclides (cf. Table III). Figure 3 shows our results together with the values of Ref. [16]. No dependence on $A_{t}$ is seen for the investigated nuclei. The weighted mean for the yield of $\left(A_{t}-1\right)$ nuclei per antiproton is $(102.2 \pm 2.4) \times 10^{-3}$ (only the statistical error). As the ${ }^{48} \mathrm{Ca}$ target was a carbonate the branching ratio for antiproton capture into $\mathrm{Ca}, \mathrm{C}$, and $\mathrm{O}$ had to be taken into account. It was assumed to be the same as that for muons. This should be a good approximation as the capture mainly depends on the configuration of the valence electrons. The capture ratios for muons were taken from Refs. [24-26]. Only after the experiment did we find out that the carbonate had absorbed $\mathrm{H}_{2} \mathrm{O}$ and hence at the time of the measurement the target contained an unknown amount of water. The composition could have been between pure $\mathrm{CaCO}_{3}$ and $\mathrm{CaCO}_{3} \cdot 6 \mathrm{H}_{2} \mathrm{O}$. In order to correct for capture into hydrogen, the capture probability relative to carbon was taken from a measurement with propane [27]. The resulting fraction of antiprotons captured in $\mathrm{Ca}$ was between $(14 \pm 2) \%$ and $(35 \pm 4) \%$. In the case of the ${ }^{104} \mathrm{Ru}$ target the metal powder was mixed with glue. In order to correct for this, a pure glue target was irradiated and the yield of residual nuclei with $Z<8$ was compared with the yield from the glued ruthenium target. The total number of antiprotons captured by ${ }^{104} \mathrm{Ru}$ was corrected by $(56 \pm 12) \%$

TABLE III. Yields $N(\bar{p}, n)$ of $\left(N_{t}-1\right)$ and $N(\bar{p}, p)$ of $\left(Z_{t}-1\right)$ nuclei, peripheral halo factor, and absolute yield of $\left(A_{t}-1\right)$ nuclei.

\begin{tabular}{|c|c|c|c|c|c|}
\hline Target & $\frac{N(\bar{p}, n)}{1000 \bar{p}}$ & $\frac{N(\bar{p}, p)}{1000 \bar{p}}$ & $\frac{N(\bar{p}, n)}{N(\bar{p}, p)}$ & $f_{\text {halo }}^{\text {periph }}$ & $\frac{N\left(A_{t}-1\right)}{1000 \bar{p}}$ \\
\hline${ }^{48} \mathrm{Ca}$ & $76(38)$ & $29(15)$ & $2.62(30)$ & $2.97(34)$ & $105(53)$ \\
\hline${ }^{100} \mathrm{Mo}$ & $66(6)$ & $23.4(15)$ & $2.82(23)$ & $3.24(26)$ & $90(8)$ \\
\hline${ }^{104} \mathrm{Ru}$ & $55(16)$ & $19(6)$ & 2.9 (4) & $3.4(5)$ & 75 (21) \\
\hline${ }^{112} \mathrm{Sn}$ & $52(9)$ & $66(11)$ & 0.79 (14) & $1.01(18)$ & $118(12)$ \\
\hline${ }^{116} \mathrm{Cd}$ & $130(31)$ & $26(9)$ & $5.0(21)$ & $5.6(23)$ & $156(55)$ \\
\hline${ }^{124} \mathrm{Sn}$ & $98(15)$ & $19.6(15)$ & $5.0(6)$ & $5.4(6)$ & $118(9)$ \\
\hline
\end{tabular}




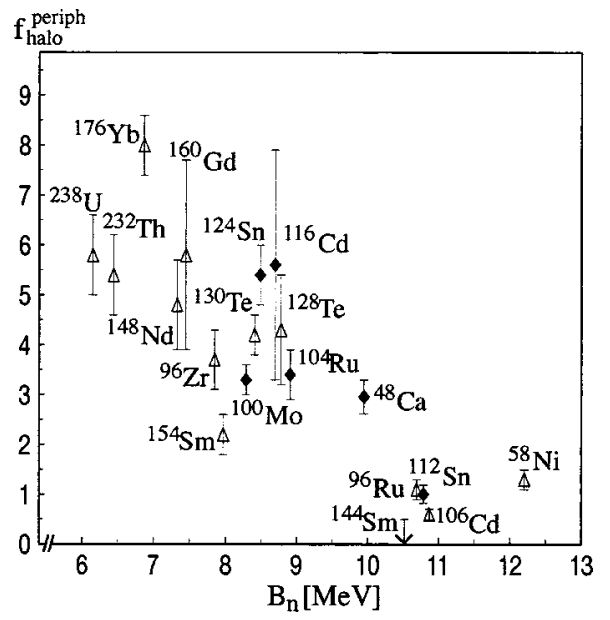

FIG. 2. Measured peripheral halo factors $f_{\text {halo }}^{\text {periph }}$ : results from the present experiment (solid diamonds) and from Ref. [16] (open triangles) versus the neutron binding energy $B_{n}$.

for antiproton capture in the glue.

\section{POPULATION OF EXCITED STATES}

Already in previous experiments on residual nuclei from $\bar{p}$ annihilation [9-11] isotopes with two metastable states were found. In most of the cases where the original nucleus had lost more than one nucleon, the population of the state with the higher spin was favored. This is presumably caused by the emission of energetic particles, which leaves the nucleus with considerable angular momentum. In contrast to this conclusion the low-spin metastable states were found to be preferentially populated in our previous study of $A_{t}-1$ nuclei [16]. This could not be corroborated by the present investigation (cf. Table IV): Three of the isomeric ratios $R_{I}$ (state with high spin to that with low spin) are larger than 1.

The calculation of $R_{I}$ is rather complicated because two effects have to be considered. First, various $\bar{p}$-atomic states contribute to antiproton annihilation, which have a different radial extension of the respective annihilation region [16] and maybe different angular momentum influx. Second, the

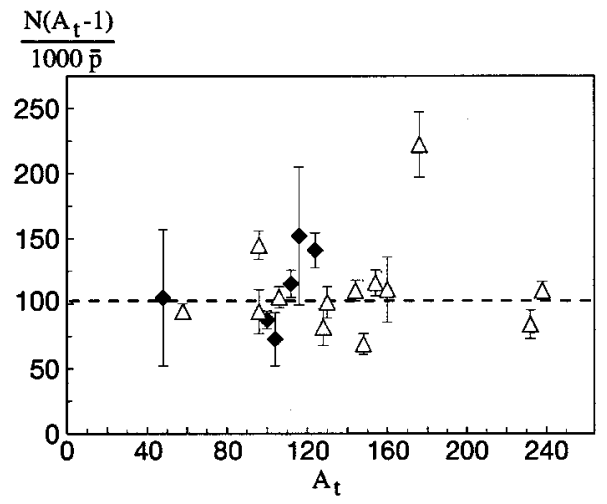

FIG. 3. Absolute number of nuclei with mass $\left(A_{t}-1\right)$ produced per $1000 \bar{p}$ as a function of the atomic mass of the target $A_{t}$ (solid diamonds). The open triangles show the data from Ref. [16]. The dashed line indicates the weighted mean.
TABLE IV. Isomeric ratios $R_{I}$ for $\left(A_{t}-1\right)$ nuclei of the measured high-spin to the low-spin state.

\begin{tabular}{lcccc}
\hline \hline Target & Isomer & Spin & Energy $(\mathrm{keV})$ & \multicolumn{1}{c}{$R_{I}$} \\
\hline${ }^{100} \mathrm{Mo}$ & ${ }^{99 g} \mathrm{Nb} /{ }^{99 m} \mathrm{Nb}$ & $\frac{9}{2}^{+} / \frac{1}{2}^{-}$ & $0 / 365.3$ & $0.379(20)$ \\
${ }^{112} \mathrm{Sn}$ & ${ }^{111 g} \mathrm{In} /{ }^{111 m} \mathrm{In}$ & $\frac{9}{2}^{+} / \frac{1}{2}^{-}$ & $0 / 536.95$ & $3.7(6)$ \\
${ }^{116} \mathrm{Cd}$ & ${ }^{115 m} \mathrm{Ag} /{ }^{115 g} \mathrm{Ag}$ & $\frac{7}{2}^{+} / \frac{1}{2}^{-}$ & $41.1 / 0$ & $0.48(24)$ \\
& ${ }^{115 m} \mathrm{Cd} /{ }^{115 g} \mathrm{Cd}$ & $\frac{11}{2}^{-} / \frac{1}{2}^{+}$ & $181.0 / 0$ & $1.4(6)$ \\
${ }^{124} \mathrm{Sn}$ & ${ }^{123 g} \mathrm{In} /{ }^{123 m} \mathrm{In}$ & $\frac{9}{2}^{+} / \frac{1}{2}^{-}$ & $0 / 327.2$ & $1.75(24)$ \\
& ${ }^{123 g} \mathrm{Sn} /{ }^{123 m} \mathrm{Sn}$ & $\frac{11}{2}^{-} / \frac{3}{2}^{+}$ & $0 / 24.6$ & $0.74(16)$ \\
\hline \hline
\end{tabular}

excited states in the $\left(A_{t}-1\right)$ nucleus populated immediately after annihilation decay either to the isomeric state or to the ground state. Thus the ratio $R_{I}$ is changed depending on the specific nuclear structure.

Prompt $\gamma$ rays emitted by the residual nucleus give insight into the excited state populated after annihilation. The large background from annihilation products and antiprotonic $\mathrm{x}$ rays makes the measurement rather difficult, especially because the probability to produce a special isotope is small. In this context the investigation of $\gamma$ rays of $\left(A_{t}-1\right)$ nuclei is most promising due to their relatively large yield (up to $10 \%)$.

For two of the investigated target nuclei, ${ }^{112} \mathrm{Sn}$ and ${ }^{124} \mathrm{Sn}$, several prompt $\gamma$ rays of $\left(A_{t}-1\right)$ nuclei from higher-lying levels could be observed. Most of these states decay within a few ns. For this investigation prompt spectra after antiproton annihilation were taken with a setup described in Refs. $[7,28]$. The corresponding $\gamma$ lines are listed in Table V.

The population of the levels was determined from the measured intensities of the lines depopulating them using the decay properties of the levels taken from Ref. [23]. The maximum excitation energy of the nuclei which could be measured was limited to about $1.3 \mathrm{MeV}$ by the energy range accepted by the HPGe detectors. The cumulative yields of the investigated levels for the four residual $\left(A_{t}-1\right)$ nuclei ${ }^{111} \mathrm{Sn},{ }^{111} \mathrm{In},{ }^{123} \mathrm{Sn}$, and ${ }^{123} \mathrm{In}$ are also presented in Table V. In those cases in which the lines of the depopulating transitions were not visible, an upper limit is given. The level of ${ }^{111} \mathrm{Sn}$ with an energy of $255 \mathrm{keV}$ has a half-life of $12.5 \mu \mathrm{s}$. Its population could not be measured with the transport system; nor could it be taken from the prompt spectrum. The yields of the ground and metastable states in Table $\mathrm{V}$ were inferred from the yields of the residual nuclei and the isomeric ratios.

The results show that during the annihilation highly excited states of the $\left(A_{t}-1\right)$ nuclei are produced with a large probability (up to $2 \%$ per level). These levels decay into the ground as well as into isomeric states. Hence, as mentioned above, the interpretation of isomeric ratios is rather difficult.

\section{COMPARISON WITH NUCLEON-DENSITY CALCULATIONS}

The experimental peripheral halo factors are compared with calculated ones in Fig. 4. While the densities derived 
TABLE V. Investigated prompt $\gamma$ lines and total population $P$ of the levels for ${ }^{111} \mathrm{Sn},{ }^{111} \mathrm{In}$, ${ }^{123} \mathrm{Sn}$, and ${ }^{123} \mathrm{In}$.

\begin{tabular}{|c|c|c|c|c|c|}
\hline & Level energy (keV) & $J^{\pi}$ & $E_{\gamma}$ & $I_{\gamma}(\%)$ & $P / 10^{3} \bar{p}$ \\
\hline \multirow[t]{8}{*}{${ }^{111} \mathrm{Sn}:$} & 0.0 & $7 / 2^{+}$ & & & $52(9)$ \\
\hline & 154.48 & $5 / 2^{+}$ & 154.48 & 100 & $13.5(10)$ \\
\hline & 254.72 & $1 / 2^{+}$ & 100.24 & 100 & - \\
\hline & 643.55 & $3 / 2^{+}$ & 489.1 & 88 & $1.07(36)$ \\
\hline & 755.36 & $5 / 2^{+}$ & 755.4 & 87 & $0.2(5)$ \\
\hline & 979.6 & $11 / 2^{-}$ & 978.6 & 100 & $1.11(31)$ \\
\hline & 1032.6 & $3 / 2^{+}$ & $\begin{array}{r}1032.6 \\
777.8\end{array}$ & $\begin{array}{l}75 \\
21\end{array}$ & 0.18 (15) \\
\hline & 1151.7 & $3 / 2^{+}, 5 / 2^{+}$ & 997.1 & 43 & 0.42 (16) \\
\hline \multirow[t]{9}{*}{${ }^{111} \mathrm{In}:$} & 0.0 & $9 / 2^{+}$ & & & $66(11)$ \\
\hline & 536.95 & $1 / 2^{-}$ & 537.22 & 100 & $14.2(8)$ \\
\hline & 802.94 & $3 / 2^{-}$ & 265.7 & 100 & $1.62(26)$ \\
\hline & 1101.80 & $5 / 2^{+}$ & 1101.18 & 96 & $0.26(26)$ \\
\hline & 1152.85 & $11 / 2^{+}$ & 1152.98 & 100 & $1.3(6)$ \\
\hline & 1187.62 & $1 / 2^{+}$ & 650.7 & 99 & $1.5(4)$ \\
\hline & 1217.51 & $5 / 2^{+}$ & 1217.4 & 91 & 0.8 (4) \\
\hline & 1280.8 & $(5 / 2)^{-}$ & 742.9 & 89 & $2.7(5)$ \\
\hline & & & 476.7 & 11 & 2.1 (ग) \\
\hline \multirow[t]{17}{*}{${ }^{123} \mathrm{Sn}:$} & 0.0 & $11 / 2^{-}$ & & & $42(9)$ \\
\hline & 24.6 & $3 / 2^{+}$ & & & $56.5(33)$ \\
\hline & 150.4 & $1 / 2^{+}$ & 125.76 & 100 & $7.9(7)$ \\
\hline & 618.81 & $(9 / 2)^{-}$ & 618.8 & 100 & $0.44(5)$ \\
\hline & 870.2 & $\left(3 / 2^{+}, 5 / 2^{+}\right)$ & 845.5 & 89 & \\
\hline & & & 719.9 & 11 & $17.9(23)$ \\
\hline & 919.8 & $(3 / 2)^{+}$ & 895.8 & 74 & 11 (11) \\
\hline & & & 769.3 & 26 & 1.1 (11) \\
\hline & 931.4 & $7 / 2^{-}$ & 931.2 & 100 & $1.9(4)$ \\
\hline & 1044.3 & $(7 / 2)^{+}$ & 1019.7 & 99 & $<0.35$ \\
\hline & 1072.1 & $(1 / 2,3 / 2)^{+}$ & 921.9 & 57 & 21 (7) \\
\hline & & & 1046.8 & 43 & $2.1 \quad(1)$ \\
\hline & 1107 & $\left(15 / 2^{-}\right)$ & 1107 & & $<0.34$ \\
\hline & 1136.3 & $(1 / 2,3 / 2,5 / 2,7 / 2)^{+}$ & 1112.0 & 60 & 41 (9) \\
\hline & & & 985.6 & 40 & 4.1 (9) \\
\hline & 1155.0 & $7 / 2^{+}$ & 1130.5 & 98 & $3.2(7)$ \\
\hline & 1194.4 & $5 / 2^{+}$ & 1169.8 & 100 & $0.50(25)$ \\
\hline \multirow[t]{6}{*}{${ }^{123} \mathrm{In}:$} & 0.0 & $9 / 2^{+}$ & & & $12.5(12)$ \\
\hline & 327.21 & $1 / 2^{-}$ & & & $7.1(9)$ \\
\hline & 698.55 & $(3 / 2)^{-}$ & 371.32 & 100 & $1.43(23)$ \\
\hline & 1027.39 & $\left(9 / 2,11 / 2,13 / 2^{+}\right)$ & 1027.45 & 100 & $<0.36$ \\
\hline & 1052.29 & $5 / 2^{+}$ & 1052.28 & 87 & $<2.09$ \\
\hline & 1137.51 & $\left(1 / 2,3 / 2,5 / 2^{-}\right)$ & 810.29 & 73 & $<0.75$ \\
\hline
\end{tabular}

from a modified Fermi gas model $[8,29]$ resulted in too large values for the peripheral neutron halo factor, results from Hartree-Fock-Bogoliubov (HFB) calculations with Skyrme forces $[8,16]$ were found to be a good description of $f_{\text {halo }}^{\text {periph }}$ for many of the measured nuclei. There are, however, some special cases, such as ${ }^{112} \mathrm{Sn}$, for which the neutron-to-proton density ratio in the nuclear periphery is badly represented.

Relativistic Hartree-Bogoliubov (RHB) calculations should be more promising, as they provide a good descrip- tion for the neutron halo of light nuclei [30] and of nuclei far from the valley of $\beta$ stability, where pairing effects are important. In order to provide a good description of the nuclear periphery the equations for the nucleons and the meson field have to be solved in coordinate space. A code to perform RHB calculations with finite-range pairing forces in coordinate-space discretization has been developed during the past years [31]. However, for the six investigated nuclei no better agreement with the measured halo factors was 


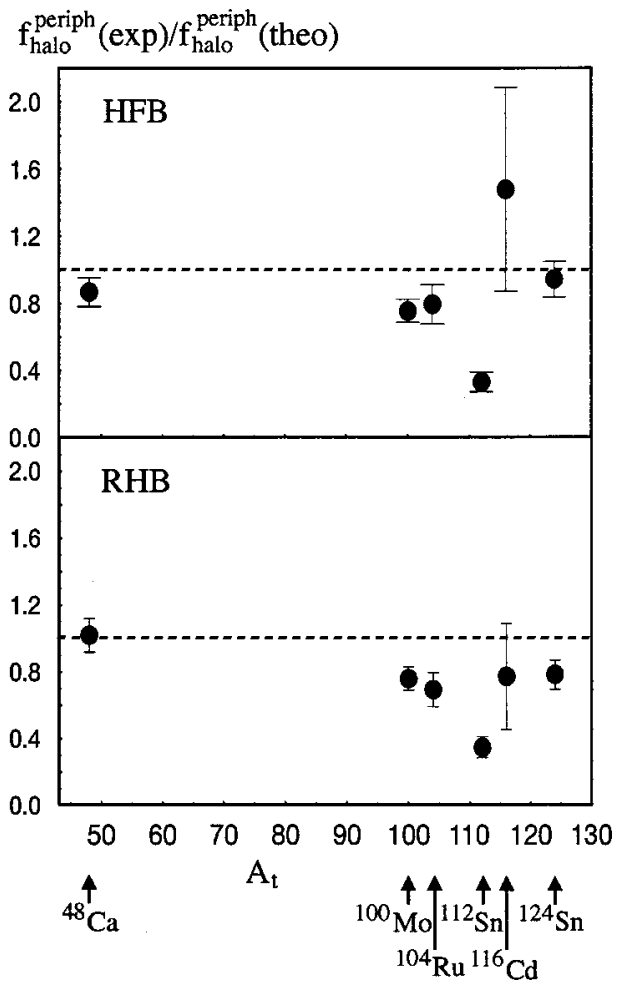

FIG. 4. Ratio of experimental to theoretical value for the peripheral halo factor as a function of the target mass $A_{t}$. Upper part: theoretical $f_{\text {halo }}^{\text {periph }}$ values from HFB calculations. Lower part: theoretical $f_{\text {halo }}^{\text {periph }}$ values from RHB calculations. The dashed lines indicate a ratio value of 1 .

found than with the HFB calculations (cf. Fig. 4).

A comparison of measured halo factors with those derived from the HFB model may be used to determine the ratio $\mathcal{R}$ of the imaginary parts of the effective scattering length of antiprotons on bound neutrons and protons. From Eq. (3) we obtain

$$
\mathcal{R}=\frac{\operatorname{Im}\left(a_{n}\right)}{\operatorname{Im}\left(a_{p}\right)}=\frac{N(\bar{p}, n) Z_{t}}{N(\bar{p}, p) N_{t}} / f_{\text {halo }}^{\text {periph }},
$$

with $N(\bar{p}, n)$ and $N(\bar{p}, p)$ taken from the experiment and $f_{\text {halo }}^{\text {periph }}$ from the HFB calculations. The results from RHB calculations were not used, as such calculations have not yet been performed for all investigated nuclei. $\mathcal{R}$ is plotted in Fig. 5 versus the target mass $A_{t}$. No dependence of $\mathcal{R}$ on the mass number $A_{t}$ of the target nuclei is evident. Especially the lightest (with $A_{t}$ around 50) and heaviest nuclei (with $A_{t}$ around 230) lead to a similar $\mathcal{R}$.

If one assumes $\mathcal{R}$ to be constant, its mean value can be deduced from the data to be $\mathcal{R}=0.50$ (7) (cf. the dashed line in Fig. 5) with a $\chi^{2}$ per data point of 13 . This value, however, is not satisfactory, as it lies well below most of the data points, due to a few very low-lying points with small uncertainties. Out of the 19 data points 15 are consistent with a constant value for $\mathcal{R}$. However, there are some striking discrepancies. This might have two explanations. Either $\mathcal{R}$ depends on some other special properties of the nuclei, or the

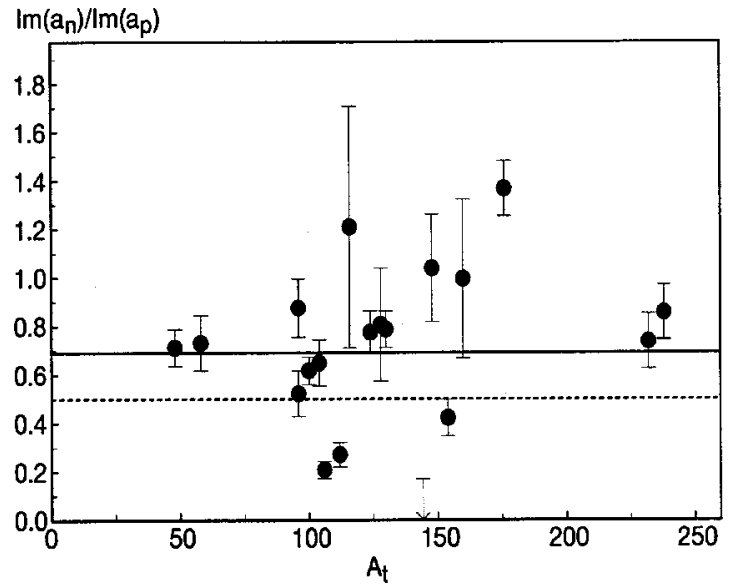

FIG. 5. Ratio $\mathcal{R}$ of imaginary parts of the effective scattering length of antiprotons on bound neutrons and protons versus the target mass $A_{t}$. The dashed line shows the value $\mathcal{R}=0.50$; the solid line, $\mathcal{R}=0.69$.

calculated values for $f_{\text {halo }}^{\text {periph }}$ are not correct for very low $\left({ }^{106} \mathrm{Cd},{ }^{112} \mathrm{Sn}\right.$, and $\left.{ }^{144} \mathrm{Sm}\right)$ or for very high $\left({ }^{176} \mathrm{Yb}\right)$ neutronto-proton density ratios. However, no correlation was found for $\mathcal{R}$ with the calculated neutron-to-proton density ratio, with the neutron excess, with proton and neutron binding energies, or with pairing energies. For ${ }^{176} \mathrm{Yb}$ the calculated ratio is too small by a factor of more than 2 [16]. This nucleus is a special case, as it is strongly deformed, and the shape of the nucleus may be complicated [32]. On the other hand, the small measured halo factors of ${ }^{106} \mathrm{Cd}$ and ${ }^{144} \mathrm{Sm}$ were not reproduced [16], and for ${ }^{112} \mathrm{Sn}$ the calculated peripheral halo factor is also too large (cf. Fig. 5).

In general, the HFB model does not predict properly the separation energies and sometimes overestimates the neutron-to-proton density ratio in the nuclear periphery. Especially for proton-rich nuclei with $f_{\text {halo }}^{\text {periph }} \leqslant 1$ the calculated ratio is more than twice as large as the measured one. For the proton-rich nuclei ${ }^{58} \mathrm{Ni}$ and ${ }^{96} \mathrm{Ru}$ with lower $Z$, on the other hand, the calculations agree with the experiment.

If the average value of $\mathcal{R}$ is calculated with only $15 \mathrm{nu}-$ clei, without the values of the four nuclei discussed above, the result is $\mathcal{R}=0.69(4)$ with a $\chi^{2}$ per data point of 2.0. This agrees well with the value of $0.63(6)$ of Refs. $[14,20]$, deduced from investigations on carbon, but it differs from the results of measurements with deuterium, $\mathcal{R}=0.82$ (4) [21], and helium, $\mathcal{R}=0.47(4)$ [22]. The line in Fig. 5 shows the mean value $\mathcal{R}=0.69$. This new result is relevant for the determination of the neutron density from antiprotonic $\mathrm{x}$ rays, as was done in [7] for ${ }^{172} \mathrm{Yb}$ and ${ }^{176} \mathrm{Yb}$ with the value $\mathcal{R}=0.82$ from Ref. [21], proposed in [8]. The analysis should be repeated with $\mathcal{R}=0.69$.

\section{SUMMARY}

The additional measurements of the peripheral halo factor described in this publication confirmed an enhanced neutronto-proton density ratio to exist in the nuclear periphery for a large number of nuclei. The strongly negative linear correla- 
tion of this factor with the neutron binding energy was also corroborated.

The results were compared with those from different nuclear models. For a large number of nuclei the agreement of the experimental data with Hartree-Fock-Bogoliubov and relativistic Hartree-Bogoliubov calculations is good. However, there are some nuclei where this agreement is very poor. From a comparison with the HFB results the ratio of the absorption strength of the antiproton on a neutron to that on a proton could be deduced.

The measured peripheral halo factors and a systematic investigation of antiprotonic $\mathrm{x}$ rays, which was published recently for $\mathrm{Yb}$ isotopes [7] and will be published soon for several other elements, provide a large number of observables for the combined analysis of the antiproton-nucleus effective scattering length and of neutron and proton densities at the nuclear surface.

The investigation of excited states of the $\left(A_{t}-1\right)$ residual nuclei of ${ }^{112} \mathrm{Sn}$ and ${ }^{124} \mathrm{Sn}$ revealed that besides isomeric states also other excited states are produced with a large probability. A dedicated study of the in-beam $\gamma$ transitions of $\left(A_{t}-1\right)$ nuclei could add new information on the antiproton annihilation process.

\section{ACKNOWLEDGMENTS}

We thank the LEAR team for providing the intense, highquality antiproton beam, Anna Stolarz of the Heavy Ion Laboratory in Warsaw, and Katharina Nacke and Peter Maier-Komor of the Technical University Munich for the target preparation, M. Aigner, H. Angerer, and G. Singer for technical assistance, and Jacek Dobaczewski for valuable discussions. Financial support by the Accelerator Laboratory of the University and the Technical University of Munich, as well as by the Polish State Committee for Scientific Research under Grant Nos. 2 P03B 04815 and 2 P03B 01615 is acknowledged. This work was also supported by the Volkswagen Foundation.
[1] C. J. Batty, E. Friedman, and A. Gal, Phys. Rep. 287, 385 (1997).

[2] F. J. Hartmann, in Electromagnetic Cascade and Chemistry of Exotic Atoms, edited by L. M. Simons et al. (Plenum Press, New York, 1990), p. 127.

[3] C. J. Batty, E. Friedman, and A. Gal, Nucl. Phys. A592, 487 (1995).

[4] Th. Köhler, P. Blüm, G. Büche, A. D. Hancock, H. Koch, A. Kreissl, H. Poth, U. Raich, D. Rohmann, G. Backenstoss, Ch. Findeisen, J. Repond, L. Tauscher, A. Nilsson, S. Carius, M. Suffert, S. Charalambus, M. Chardalas, S. Dedoussis, H. Daniel, T. von Egidy, F. J. Hartmann, W. Kanert, G. Schmidt, J. J. Reidy, M. Nicholas, and A. Wolf, Phys. Lett. B 176, 327 (1986).

[5] A. Kreissl, A. D. Hancock, H. Koch, Th. Köhler, H. Poth, U. Raich, D. Rohmann, A. Wolf, L. Tauscher, A. Nilsson, M. Suffert, M. Chardalas, S. Dedoussis, H. Daniel, T. von Egidy, F. J. Hartmann, W. Kanert, H. Plendl, G. Schmidt, and J. J. Reidy, Z. Phys. A 329, 239 (1988).

[6] W. Kanert, F. J. Hartmann, H. Daniel, E. Moser, G. Schmidt, T. von Egidy, J. J. Reidy, M. Nicholas, M. Leon, H. Poth, G. Büche, A. D. Hancock, H. Koch, Th. Köhler, A. Kreissl, U. Raich, D. Rohmann, M. Chardalas, S. Dedoussis, M. Suffert, and A. Nilsson, Phys. Rev. Lett. 56, 2368 (1986).

[7] R. Schmidt, F. J. Hartmann, T. von Egidy, T. Czosnyka, J. Iwanicki, J. Jastrzȩbski, M. Kisieliński, P. Lubiński, P. Napiorkowski, L. Pieńkowski, A. Trzcińska, R. Smolańczuk, S. Wycech, B. Kłos, K. Gulda, W. Kurcewicz, and E. Widmann, Phys. Rev. C 58, 3195 (1998).

[8] S. Wycech, J. Skalski, R. Smolańczuk, J. Dobaczewski, and J. R. Rook, Phys. Rev. C 54, 1832 (1996).

[9] E. F. Moser, H. Daniel, T. von Egidy, F. J. Hartmann, W. Kanert, G. Schmidt, Ye. S. Golubeva, A. S. Iljinov, M. Nicholas, and J. J. Reidy, Z. Phys. A 333, 89 (1989).

[10] T. von Egidy, H. Daniel, F. J. Hartmann, W. Kanert, E. F. Moser, Ye. S. Golubeva, A. S. Iljinov, and J. J. Reidy, Z. Phys. A 335, 451 (1990).
[11] J. Jastrzȩbski, W. Kurcewicz, P. Lubiński, A. Grabowska, A. Stolarz, H. Daniel, T. von Egidy, F. J. Hartmann, P. Hofmann, Y. S. Kim, A. S. Botvina, Ye. S. Golubeva, A. S. Iljinov, G. Riepe, and H. S. Plendl, Phys. Rev. C 47, 216 (1993).

[12] J. Jastrzȩbski, H. Daniel, T. von Egidy, A. Grabowska, Y. S. Kim, W. Kurcewicz, P. Lubiński, G. Riepe, W. Schmid, A. Stolarz, and S. Wycech, Nucl. Phys. A558, 405c (1993).

[13] R. C. Barret and D. F. Jackson, Nuclear Sizes and Structure (Clarendon Press, Oxford, 1977).

[14] W. M. Bugg, G. T. Condo, E. L. Hart, H. O. Cohn, and R. D. McCulloch, Phys. Rev. Lett. 31, 475 (1973).

[15] P. Lubiński, J. Jastrzȩbski, A. Grochulska, A. Stolarz, A. Trzcińska, W. Kurcewicz, F. J. Hartmann, W. Schmid, T. von Egidy, J. Skalski, R. Smolańczuk, S. Wycech, D. Hilscher, D. Polster, and H. Rossner, Phys. Rev. Lett. 73, 3199 (1994).

[16] P. Lubiński, J. Jastrzȩbski, A. Trzcińska, W. Kurcewicz, F. J. Hartmann, W. Schmid, T. von Egidy, R. Smolańczuk, and S. Wycech, Phys. Rev. C 57, 2962 (1998).

[17] C. J. Batty, in Antiproton-Nucleon and Antiproton-Nucleus Interactions, edited by F. Bradamante et al. (Plenum Press, New York, 1990), p. 251.

[18] M. Leon and R. Seki, Phys. Lett. 48B, 173 (1974).

[19] C. J. Batty, Nucl. Phys. A372, 433 (1981).

[20] M. Wade and V. G. Lind, Phys. Rev. D 14, 1182 (1976).

[21] R. Bizzarri, P. Guidoni, F. Marcelja, F. Marzano, E. Castelli, and M. Sessa, Nuovo Cimento A 22, 225 (1974).

[22] F. Balestra et al., Nucl. Phys. A491, 572 (1989).

[23] NNDC Online Data Service, Brookhaven National Laboratory, telnet address: bnlnd2.dne.bnl.gov

[24] J. D. Knight, C. J. Orth, M. E. Schillaci, R. A. Naumann, H. Daniel, K. Springer, and H. B. Knowles, Phys. Rev. A 13, 43 (1976).

[25] T. von Egidy and F. J. Hartmann, Phys. Rev. A 26, 2355 (1982).

[26] T. von Egidy, D. H. Jakubassa-Amundsen, and F. J. Hartmann, Phys. Rev. A 29, 455 (1984). 
[27] W. T. Pawlewicz, C. T. Murphy, J. G. Fetkovich, T. Dombeck, M. Derrick, and T. Wangler, Phys. Rev. D 2, 2538 (1970).

[28] R. Schmidt, F. J. Hartmann, B. Ketzer, T. von Egidy, T. Czosnyka, J. Jastrzȩbski, M. Kisieliński, P. Lubiński, P. Napiorkowski, L. Pieńkowski, A. Trzcińska, B. Kłos, R. Smolańczuk, S. Wycech, K. Gulda, W. Kurcewicz, and E. Widmann (unpublished).

[29] J. Dobaczewski, H. Flocard, and J. Treiner, Nucl. Phys. A422,
103 (1984)

[30] W. Pöschl, D. Vretenar, G. A. Lalazissis, and P. Ring, Phys. Rev. Lett. 79, 3841 (1997).

[31] W. Pöschl, D. Vretenar, and P. Ring, Comput. Phys. Commun. 103, 217 (1997).

[32] T. Cooper, W. Bertozzi, J. Heisenberg, S. Kowalski, W. Turchinetz, C. Williamson, L. Cardman, S. Fivozinsky, J. Lightbody, Jr., and S. Penner, Phys. Rev. C 13, 1083 (1976). 